\title{
Heading Measurement based on Ultrasonic and Magnetic Compass
}

\author{
BaoJiang Sun ${ }^{1, a}$, Yue $\mathrm{Xu}^{2, \mathrm{~b}}$ \\ ${ }^{122}$ Shandong Polytechnic University, Jinan 250300, Shandong, China \\ asbj@vip.sina.com..calmy1@163.com.
}

Keywords: Heading Measure, Kalman Filter, Magnetic Compass, Ultrasonic

\begin{abstract}
Describes briefly ultrasonic positioning system (UPS) and digital magnetic compass(DMC) heading measurement principle,analyzed the advantages and disadvantages of each option. To improve the accuracy of the heading measurement, As the theoretical basis of adaptive Kalman filter, designed a kind of ups and dmc integrated navigation system. Based on both real measurement data, made a simulation experiment and confirmed the feasibility of the navigation system.
\end{abstract}

\section{Introduction}

There is no error accumulation when using UPS to measure the absolute heading of the mobile carrier, heading output is almost free from outside interference. However, due to the ultrasonic distance measurement errors exist and the poor real-time measure, to improve the accuracy of ultrasonic measurement, it needs to achieve the information integration with other equipments. DMC has high accuracy and good real-time in the measurement of heading,but it is highly vulnerable to the effects of the external magnetic field so as to produce deviation.In order to improve the accuracy of heading measurement, this paper make use of two methods of complementarity to adaptive kalman filter for the bridge to design an integrated structure of two methods , and did simulation experiment. The results show that this program is effective to improve the accuracy of the heading measurement.

\section{Heading measurement based on UPS}

UPS is composed by a plurality of ultrasonic sensors in accordance with certain rules ranging system, has been extensive studied and applied in local positioning of the mobile carrier.UPS generally consists of a plurality of ultrasonic receiver and an ultrasonic transmitter,the transmitter is mounted on the mobile carrier. The current coordinates of the moving carrier is calculated by measuring the distance of the transmitter with the receiver,as in Figure 1,through the distance between the receiving end of the known coordinates $P_{1}, P_{2}, P_{3}$, and the transmitter $D$, according to three goals intersection positioning principle, the $D$ point coordinates $\left(x_{D}, y_{D}, z_{D}\right)$ can be obtained[1].

Learning from the heading measurement technology in GPS navigation system, we place an ultrasonic transmitter in the mobile carrier front and rear ends, as in Figure 2.The connection distance of the center of the two transmitters is $d$, assuming at one point the carrier heading angle is $\alpha$, the estimated value of two points coordinates $\mathrm{A}, \mathrm{B}$ is $\left(x_{A}, y_{A}, z_{A}\right),\left(x_{B}, y_{B}, z_{B}\right)$ measured by UPS.As the carrier movement is in the two-dimensional flat space, regardless the pitch angle and roll angle. Clearly, the following equation established,

$$
\left\{\begin{array}{l}
x_{B}=x_{A}+d \cos \alpha \\
y_{B}=y_{B}+d \sin \alpha
\end{array}\right.
$$

Through this equation we can get the solution to the carrier heading angle $\alpha$ [2][3]. 


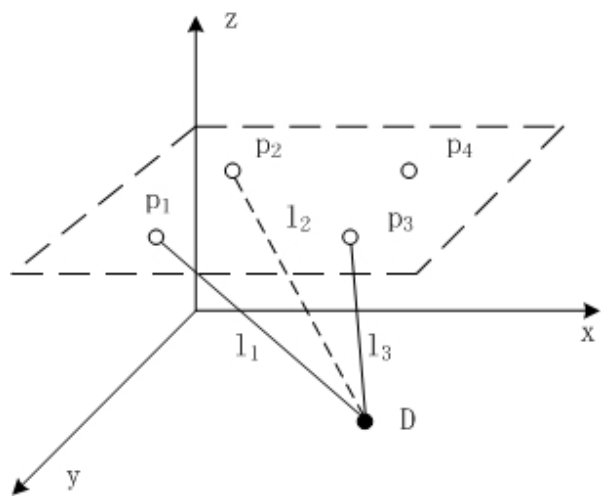

Fig.1 ultrasonic positioning principle

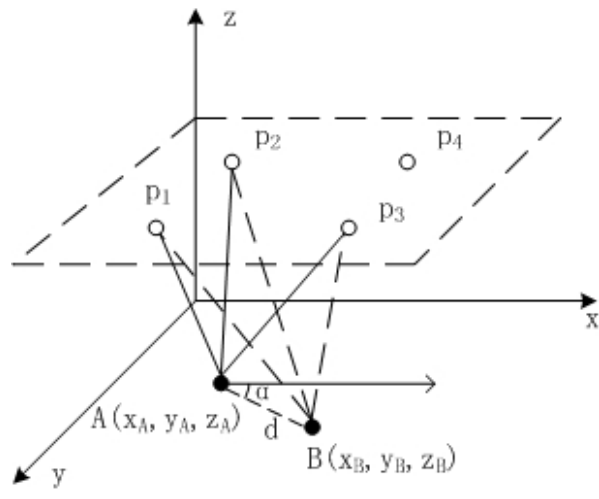

Fig.2 Heading angle measurement

\section{DMC}

The magnetic compass is usually composed of a three-axis magnetoresistive sensor, a triaxial accelerometer and signal conditioning circuit and reset degaussing circuit. Magnetic compass measurement principle is based on magnetic field component of two perpendicular magnetic sensing axial that has been changed into a level, and calculate the resultant vector direction that Magnetic north.Then find the heading of the carrier[4]. Through the accelerometer sensors measuring carrier and horizontal plane angle, so as to determine the carrier pitching angle and roll angle. A typical structure of the magnetic compass,as in figure 3.

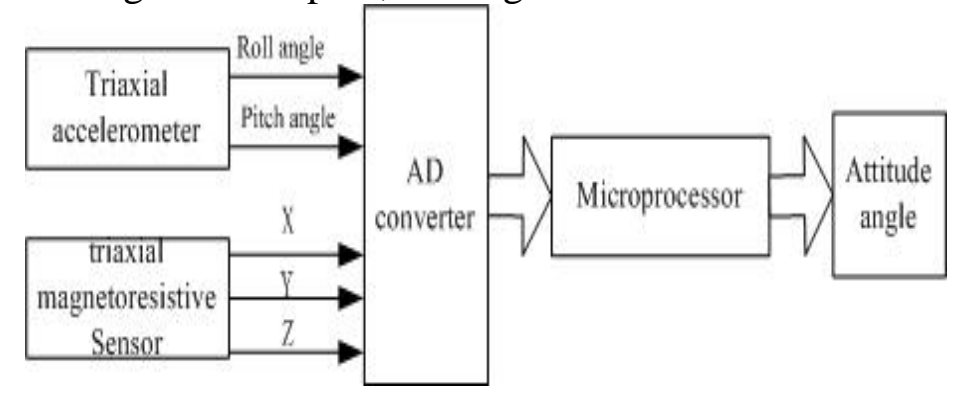

Fig.3 The structural principle of magnetic compass

Mobile carrier herein is limited to two-dimensional plane, so we only study the magnetic compass heading angle output[5].

\section{The integrated structure of UPS and DMC}

Kalman filtering is mainly applied to the linear state projected and observed values both exist noise, weighted projected and observed values, obtain the optimal estimate of the state. In the integrated system, the first step prediction can be obtained by UPS measured values with the optimal estimation of the last cycle. Its observed value directly measured by a DMC,establishing kalman filtering equation.The measured values of the UPS is treated as a control input, observed value is discriminanted from the measured values of DMC by UPS. Principle of system component as showed in Figure 4.

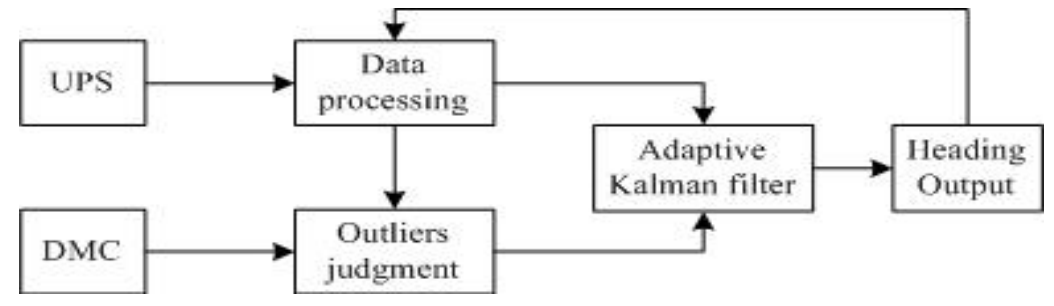

Fig.4 Principle of system component 


\section{Adaptive Kalman filter with unknown time-varying noise statistics system}

For linear discrete dynamic system

$$
\begin{gathered}
x(t+1)=\Phi(t) x(t)+w(t) \\
y(t)=H(t) x(t)+v(t)
\end{gathered}
$$

Wherein $x(t)$ is a state vector $n \times 1, y(t)$ is a observation vector $m \times 1, \Phi(t), H(t)$ are known $n \times m, m \times n$ matrix, $w(t)$ and $v(t)$ are independent with time-varying mean and covariance matrix of the normal white noise:

$$
\begin{aligned}
& E[w(t)]=q(t) \operatorname{Cov}[w(t), w(i)]=Q(t) \delta_{t i} \\
& E[v(t)]=r(t) \quad \operatorname{Cov}[v(t), v(i)]=R(t) \delta_{t i}
\end{aligned}
$$

Wherein $E$ is the mean number, Cov is the covariance number, $\delta_{t i}$ is Kronecker function[6]. Time-varying noise statistics estimators:

$$
\begin{aligned}
\hat{\mathrm{q}}(t+1)=\left(1-d_{t}\right) \hat{\mathrm{q}}(t)+d_{t}[\hat{x}(t+1 \mid t+1)-\Phi(t) \hat{x}(t \mid t)] \\
\hat{Q}(t+1)=\left(1-d_{t}\right) \hat{Q}(t)+d_{t}\left[K(t+1) \mathcal{\varepsilon}(t+1) \varepsilon^{\tau}(t+1) K^{\tau}(t+1)\right. \\
\left.+P(t+1 \mid t+1)-\Phi(t) P(t \mid t) \Phi^{\tau}(t)\right] \\
\hat{R}(t+1)=\left(1-d_{t}\right) \hat{R}(t)+d_{t}\left[\varepsilon(t+1) \varepsilon^{\tau}(t+1)\right. \\
\left.-H(t+1) P(t+1 \mid t) H^{\tau}(t+1)\right]^{-1} \\
\hat{r}(t-1)=\left(1-d_{t}\right) \hat{r}(t)+d_{t}[y(t+1)-H(t+1) \hat{x}(t+1 \mid t)] \\
d_{t}=(1-b) /\left(1-b^{t+1}\right)
\end{aligned}
$$

Adaptive Kalman filter:

$$
\begin{gathered}
\hat{x}(t+1 \mid t+1)=\hat{x}(t+1 \mid t)+K(t+1) \mathcal{E}(t+1) \\
\hat{x}(t+1 \mid t)=\Phi(t) \hat{x}(t \mid t)+\hat{q}(t) \\
\varepsilon(t+1)=y(t+1)-H(t+1) \hat{x}(t+1 \mid t)-\hat{r}(t) \\
K(t+1)=P(t+1 \mid t) H^{\tau}(t+1)[H(t+1) P(t+1 \mid t) \\
\left.H^{\tau}(t+1)+\hat{R}(t)\right]^{-1} \\
P(t+1 \mid t)=\Phi(t) P(t \mid t) \Phi^{\tau}(t)+\hat{Q}(t) \\
P(t+1 \mid t+1)=[I-K(t+1) H(t+1)] P(t+1 \mid t)
\end{gathered}
$$

Wherein $\tau$ is a permutation number, $I$ is a unit matrix, $b$ is the forgetting factor. The initial condition is $\hat{x}(0 \mid 0), P(0 \mid 0) ; \hat{r}(0), \hat{R}(0) ; \hat{q}(0), \hat{Q}(0)$.

Algorithm (1)- (5),(6)-(11),constitute the improvement of Sage and Husa adaptive Kalman filter[7][8]. 


\section{Simulation}

Mounting ultrasonic positioning equipment and DMC in a mobile robot ,let it walk on the track which has 50 degrees of heading angle. Record the measurement error both, as the simulation input. UPS and DMC sampling period were $0.2 \mathrm{~s}$ and $0.5 \mathrm{~s}$.Heading discrete system is expressed as

$$
\begin{gathered}
x(t+1)=\Phi(t) x(t)+w(t) \\
y(t)=H(t) x(t)+v(t)
\end{gathered}
$$

Wherein, $\Phi=1, H=1 . x(t)$ is heading state, $w(t)$ is UPS error, $y(t)$ is measurement value of DMC, $v(t)$ is $y(t)$ measurement noise. Take the initial value $b=0.98, \hat{x}(0 \mid 0)=50.3, \varepsilon(0)=0, P(0 \mid 0)=0, \hat{r}(0)=0, \hat{R}(0)=0.001, \hat{q}(0)=0.02, \hat{Q}(0)=0.001$. Simulation results showed in Figure 5.

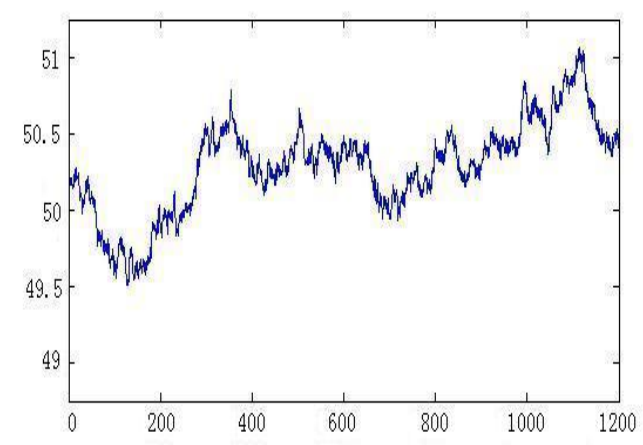

measurement value of DMC

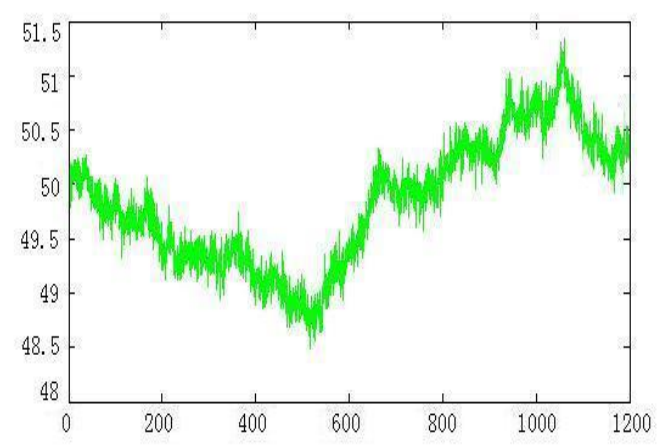

estimates of UPS

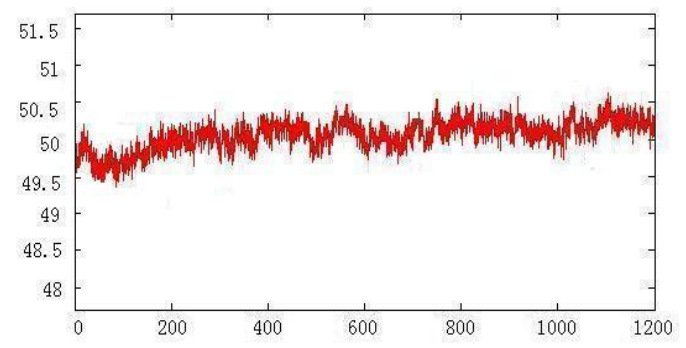

filtered value

Fig.5 Simulation effect

From the diagram we can see, separate measurement using UPS and DMC exists a great error,but the average error using adaptive Kalman filter is much lower than the first two methods, heading measurement results are nearly to the true value.

\section{Conclusion}

This paper analyzes the UPS and DMC in heading measurement characteristics, pointed out their respective advantages and disadvantages, use Kalman filter algorithm, puts forward a new heading measurement program and realizes the integration of the two complementary. Improved the measurement accuracy of UPS and DMC.

\section{References}

[1]Z.K.SUN. Single multi-base active passive positioning technology, National Defence Industry Press,1996,ch.2 
[2]H.N.WANG. Navigation Principles and Applications, Science Press,2003.

[3]M.Y.CUI,Z.L.DONG,Y.T.TIAN, Study on Laser Global Location System for Mobile Robot, Journal of Systems Engineering and Electronics,vol.24,no.11,pp.73-75,2001.

[4]J.Luo.Research on the modularized intelligent magnetic heading system. Nanjing University of Aeronautics and Astronautics,2003,pp.7-12.

[5]G.Q.Liu,Y.SH.Xue.Application of magnetic heading sensor in UAV flight control system,Journal of Transducer Technology,vol.22,no.12,pp.54-56,2003.

[6] Zi.Li.DENG, Optimal Estimation Theory and Its Applications, Harbin Institute of Technology Press,2005,pp.134-140.

[7]Duanyubo, Optimal filtering theory and its Application, Harbin Institute of Technology Press,1994,pp.64-69

[8] Sage AP,Husa GW,Adaptive filtering with unknown prior statistics.Joint Automatic Control Confer-ence,1969,pp.760-774. 